\title{
Significance of the changes occurring in the levels of interleukins, SOD and MDA in rat pulmonary tissue following exposure to different altitudes and exposure times
}

\author{
GU YUHAI* and ZHAO ZHEN* \\ Department of Respiratory Medicine, Qinghai Provincial People's Hospital, Xining, Qinghai 810007, P.R. China
}

Received May 28, 2015; Accepted July 1, 2015

DOI: $10.3892 /$ etm.2015.2604

\begin{abstract}
The aim of the present study was to investigate the changes occurring in the levels of interleukin, superoxide dismutase (SOD) and malondialdehyde (MDA) in rat lung tissue at different altitudes and times, and to determine the significance of such changes. A total of 88 Wistar rats were randomly divided into 3 groups as follows: the control group [low altitude (LA), 1,500 m; $\mathrm{n}=8$ ], the moderate altitude group (MA group, 2,260 m; $\mathrm{n}=40$ ) and the high altitude group (HA group, 5,000 m; $\mathrm{n}=40)$. The moderate and high altitude groups were subdivided into the 1, 3, 7, 15 and 30 day groups (MA1, 3, 7, 15, 30 and HA1, 3, 7, 15, 30; n=8). The levels of interleukins (IL-6, IL-8 and IL-10) in the rat lung tissue were determined by ELISA. The WST-1 Cell Proliferation Assay kit and total bile acids assay were used to determine the activity levels of SOD and the content of MDA, respectively. Compared to the control group, the levels of IL-6/IL-8/IL-10 were higher in the MA1 group; however, no significant differences were observed between the other MA subgroups. In addition, no significant differences were detected in SOD activity and the MDA content in the MA subgroups. The levels of IL-6/IL- 8 in all the HA subgroups were higher compared to those of the control group, and with the passing of time, the levels of IL-6/IL- 8 decreased, but were still higher than those of the control group. However, the level of IL-10 decreased with the passing of time, and was lower in all the HA subgroups compared to the control group. With the passing of time, SOD activity decreased, and the MDA content gradually increased. On the whole, the findings of this study indicate that hypoxia due to high altitude induces lung inflam-
\end{abstract}

Correspondence to: Dr Gu Yuhai, Department of Respiratory Medicine, Qinghai Provincial People's Hospital, 2 Gonghe Lu, Xining, Qinghai 810007, P.R. China

E-mail: qhguyuhai@163.com

*Contributed equally

Key words: rat pulmonary tissue, interleukin, superoxide dismutase, malondialdehyde, different altitudes and exposure times mation and oxidative damage, which subsequently causes severe damage to lung tissue.

\section{Introduction}

Hypoxia is a key adverse effect which occurs following exposure to high altitude, leading to lung airway inflammation and lung tissue injury. Interleukins (ILs) and oxidative stress play an important role in this process.

IL-6 is produced by various immune and non-immune cells, including vascular endothelial cells, monocytes/macrophages, keratinocytes, fibroblasts, T lymphocytes and B lymphocytes. The major biological function of IL- 6 is to promote the synthesis of acute proteins in the liver, as well as to induce the proliferation and differentiation of, and antibody secretion from B lymphocytes. During inflammation, IL-6 functions as an endogenous pyrogen and enhances the tumor-killing activity of cytotoxic T cells and natural killer (NK) cells (1).

IL-8 is a cytokine originating from various cells, such as monocytes, fibroblasts, endothelial cells, hepatocytes, epithelial cells and T lymphocytes. IL-8 has no species-specific activity, and its functions include the chemotaxis and activation of neutrophils, the chemotaxis of basophils, $\mathrm{T}$ lymphocytes and other inflammatory cells, and it also plays a role in angiogenesis (2).

IL-10 has multiple biological activities, and is important to the functions of thymus cells, T cells, B cells, NK cells, monocytes, macrophages, mast cells, neutrophils and eosinophils. The physiological function of IL-10 is to inhibit potent specific and non-specific immune reactions and subsequent tissue injury, and to induce immune tolerance. IL-10 inhibits the synthesis and release of pro-inflammatory mediators, as well as the release of chemokines by neutrophils $(3,4,19)$.

Superoxide dismutase (SOD) is an important antioxidant that can effectively eliminate superoxide anion and protect cells from oxidative damage. Ischemia and anoxia lead to an imbalance in the metabolism of free radicals. With the accumulation of free radicals, the increased production of lipid peroxides and a significant reduction in SOD levels due to depletion, resistance to oxidative damage is decreased, leading to cellular and even organ damage (5). Malondialdehyde (MDA) is the stable metabolite of lipid peroxidation. Therefore, the level of MDA can reflect the level of the free radicals in tissue and lipid peroxi- 
dation due to free radicals, and may indirectly reflect cellular damage (6).

It has been reported that the expression levels of the pro-inflammatory cytokines, IL-6, IL-8, and those of the anti-inflammatory cytokine, IL-10, are increased following exposure to hypoxia. The activity of the antioxidant SOD is decreased, and the levels of the lipid peroxide, MDA, are increased (7-11). However, the limitations of the abovementioned is that they did not perform combined, systemic and longitudinal observations and comparisons.

To the best of our knowledge, research on ILs and on the expression of oxidative stress markers in lung tissue and the changes occurring in the levels of these markers at different altitudes and exposure times is limited. Thus, in the present study, we used exposure to low altitude conditions as the control to detect the expression levels of ILs, SOD and MDA in rat lung tissue and to observe the changes occurring in the levels of these markers following exposure to middle and high altitude conditions at different exposure times. We also aimed to determine the signifiance of these changes. The findings of our study may provide a theoretical basis for the prevention and treatment of acute and chronic pulmonary injury in a hypoxic plateau environment.

\section{Materials and methods}

Animals. A group of 88 clean grade male Wistar rats were randomly divided into 3 groups as follows: the control group [low altitude (LA), Lanzhou, 1,500 m; $\mathrm{n}=8$ ), the middle altitude group (MA group, Xining, 2,260 m; n=40) and the high altitude group (HA group, low pressure oxygen chamber, simulation of 5,000 $\mathrm{m}$ above sea level; $\mathrm{n}=40$ ). The MA and HA groups were further divided according to the different observation times into the $1,3,7,15,30$ day groups (namely MA1, MA3, MA7, MA15 and MA30; HA1, HA3, HA7, HA15 and HA30), with 8 rats in each subgroup. The living conditions and diet were basically the same for all animals apart from the exposure to different altitudes. The control animals were immediately collected in Lanzhou, China, while the other 2 groups of experimental animals were transferred from Lanzhou to Xining (China). The animals in the MA group were collected in Xining at different exposure times and those in the HA group were placed in a hypobaric chamber with a simulated altitude of $5,000 \mathrm{~m}$ above sea level, and the animals were collected at the different specified time points in the low pressure oxygen cabin. The animal experimental procedures were approved by the Ethics Committee of Qinghai Provincial People's Hospital (Xining, China).

Instruments and reagents. The low pressure oxygen chamber was obtained from Guizhou Fenglei Aviation Armament Co., Ltd., (Anshun, China; model, DYC-3000; volume, $8 \times 3 \times 3 \mathrm{~m})$, the automatic blood cell analyzer was from Shenzhen Mindray Bio-Medical Electronics Co. Ltd., (Shenzhen, China), the microtome for paraffin embedding (Leica RM 2265) was from (Leica Microsystems, Wetzlar, Germany), the inverted microscope was obtained from Olympus (Tokyo, Japan), the constant temperature water bath was purchased from (HH-S11-1 type; Beijing Xinnuo Lihua Instrument Co., Ltd, Beijing, China), the iMark/xMark instrument for measuring absorbance was from Bio-Rad (Hercules,
CA, USA), the IL-6, IL-8, IL-10 kits were purchased from USCN Life Science Inc. Wuhan (Wuhan, China) and the SOD and MDA kits were purchased from Nanjing Jiancheng Biological Co. (Jiangsu, China).

Research methods. According to the different time points of the experimental design, $5 \mathrm{ml}$ venous blood were collected from the rats in all the groups using EDTAK2 as an anticoagulant. An automatic blood cell analyzer was used for the determination of the blood hemoglobin (HGB) count, in strict accordance with the manufacturer's instructions. The rats were anesthetized with $20 \%$ urethane and the lung tissue was removed following dissection; tissue from the middle lobe of the right lung was used for pathological sections, and lung tissue morphology was observed under a microscope. The remaining lung tissue was homogenized and the levels of IL-6, IL-8, IL-10 levels were determined by enzyme-linked immunosorbent assay (ELISA) in the homogenates. The WST-1 Cell Proliferation Assay kit was used to detect SOD activity, while the content of MDA was determined by total bile acids colorimetric assay as described below:

Measurement of hemoglobin $(H G B)$ levels. Venous blood was taken from the rats and the HGB count was measured using an automatic blood cell analyzer (BC-2300; Mairui Biotec, Wuhan, China) following the manufacturer's instructions.

Lung tissue morphological analysis. The rats were anesthetized with $20 \%$ urethane and the lung tissue was removed following dissection. A small section of tissue from the middle lobe of the right lung was fixed in $4 \%$ paraformaldehyde, routinely embedded in paraffin, sectioned, and stained with hematoxylin and eosin ( $\mathrm{H} \& \mathrm{E})$, and lung tissue morphology was observed under a microscope (Olympus CH-2; Olympus Optical Co., Ltd., Tokyo, Japan).

Measurement of the levels of IL-6, IL-8 and IL-10 by ELISA. A total of $500 \mu \mathrm{g}$ lung tissue was homogenized by $1 \mathrm{X}$ phosphate buffered saline (PBS). The total protein of rat lung tissue was measured using a BCA Protein Assay kit (Pierce, Springfield, IL, USA), and the levels of IL-6, IL-8 and IL-10 were detected using a ELISA kit (USCN Life Science Inc., Los Angeles, CA, USA) in the homogenates following the manufacturer's instructions.

Measurement of SOD activity and MDA content. The WST-1 Cell Proliferation Assay kit was used to detect SOD activity, while the content of MDA was determined using a total bile acids colorimetric assay in the homogenates according to the manufacturer's instructions (Jiancheng Biotech Ltd., Nanjing, China).

$H \& E$ staining. The freshly acquired lung tissue was cut into small sampes with a size of $1 \mathrm{~mm}^{3}$, which were then fixed in $4 \%$ paraformaldehyde solution, dehydrated in a series concentration of alcohol, washed with xylol and embedded in paraffin. The paraffin-embedded tissue samples were then sectioned, and the sections were washed with distilled water, and placed on a clean and oil-free slide. They were then heated with a spirit lamp and were then incubated at room temperature for 3-7 days. Subsequently, the sections were dewaxed by 
dipping them first in xylol twice (for 15 min each), and then in $100 \%$ alcohol (twice), 95\% alcohol, 75\% alcohol, 50\% alcohol and distilled water (for 3-5 min eachy). The sections were then stained in hematoxylin for 10-30 min and washed with tap water for $15 \mathrm{~min}$. The sections became blue in color after washing. For color separation, the sections were decolored in $1 \% \mathrm{HCl}$-alcohol $(\mathrm{HCl}: 70 \%$ alcohol $=1: 100$, v/v) until the sections became light red. Subsequently, counterstaining was carried out with $0.5 \%$ eosin-alcohol (eosin $0.5 \mathrm{~g}+95 \%$ alcohol $100 \mathrm{ml}$ ) for $2-5 \mathrm{~min}$. The ultrastructure (x40 and x400 magnification) of the lung tissues was then observed under a microscope and the differences between these samples were analyzed.

Statistical analysis. Statistical analysis was performed using SPSS 17.0 software. Data are presented as the means \pm SD; comparisons between multiple groups was made by one-way ANOVA and comparisons 2 groups were made using the least significant difference (LSD) test. A value of $\mathrm{P}<0.05$ was considered to indicate a statistically significant difference.

\section{Results}

Animal model. The body weight measurements of the rats and the HGB count in the different groups are presented in Table I. The activity level and food intake were decreased in the rats in the HA group following entry into the low oxygen chamber. Consequently, the rate of increase in body weight was lower than the other groups. The HGB count in the HA group gradually increased with the passing of time, and was significantly higher compared to the control group and MA group. The difference was statistically significant $(\mathrm{P}<0.05)$, indicating that the model of high-altitude hypoxia had been successfully created by simulation at an altitude of 5,000 $\mathrm{m}$ above sea level in a low pressure oxygen cabin. Thus, this model may be used for future research.

Levels of IL-6/IL-8/IL-10 in the lung tissue of rats in the different groups. As regards the MA group, the levels of IL-6/IL-8/IL-10 were higher in the MA1 subgroup compared to the control group $(\mathrm{P}<0.05)$, whereas no significant differences in these levels were observed between the other MA subgroups and the control group ( $\mathrm{P}>0.05$; Table II).

As regards the HA group, the levels of IL-6/IL-8 in all the HA subgroups were higher compared to those of the control group $(\mathrm{P}<0.05)$; these levels decreased with the passing of time, but were still higher than those of the control group $(\mathrm{P}<0.05)$. However, the levels of IL-10 decreased with the passing of time, and were lower compared to those of control group and the MA group $(\mathrm{P}<0.05$; Table II).

Lung tissue SOD activity and MDA levels in the rats in the different groups. As regards the MA group, no significant differences in SOD activity and the MDA content were observed between all the MA subgroups and the control group (Table III).

As regards the HA group, when compared to the control group and with the passing of time, SOD activity decreased, and the MDA content gradually increased $(\mathrm{P}<0.05$; Table III).

Pathological observation of the lung tissue sections from the rats in the different groups. The lung tissue sections obtained
Table I. Changes in body weight and HGB count in the rats from the different groups.

\begin{tabular}{lll}
\hline Groups & Body weight $(\mathrm{g})$ & \multicolumn{1}{c}{ HGB $(\mathrm{g} / \mathrm{l})$} \\
\hline LA & $159.25 \pm 3.73$ & $137.25 \pm 18.22$ \\
MA1 & $160.00 \pm 4.81$ & $136.25 \pm 5.39$ \\
MA3 & $175.63 \pm 5.95$ & $138.75 \pm 9.71$ \\
MA7 & $189.75 \pm 15.17$ & $137.50 \pm 14.65$ \\
MA15 & $228.38 \pm 7.09$ & $141.13 \pm 7.85$ \\
MA30 & $316.50 \pm 13.38$ & $138.38 \pm 14.15$ \\
HA1 & $163.50 \pm 7.27$ & $141.00 \pm 8.57$ \\
HA3 & $169.13 \pm 3.60$ & $157.50 \pm 11.31^{\mathrm{a}, \mathrm{b}}$ \\
HA7 & $188.00 \pm 3.02$ & $187.13 \pm 16.78^{\mathrm{a}-\mathrm{c}}$ \\
HA15 & $209.75 \pm 9.38$ & $213.75 \pm 32.06^{\mathrm{a}-\mathrm{d}}$ \\
HA30 & $237.50 \pm 12.11$ & $236.00 \pm 10.86^{\mathrm{a}-\mathrm{e}}$ \\
\hline
\end{tabular}

${ }^{\mathrm{a}-\mathrm{e}}$ Compared with the LA, HA1, HA3, HA7 and HA15 group, respectively. Data are the means $\pm \mathrm{SD}$ ( $\mathrm{n}=8$ per group). $\mathrm{P}<0.05$ for all. $\mathrm{HGB}$, hemoglobin; LA, low altitude; HA, MA, middle altitude; HA, high altitude; MA and HA1, 2, 7, 15, 30 represent the MA and HA subgroups at $1,3,7,15$ and 30 days, respectively.

Table II. Levels of IL-6/IL-8/IL-10 in the lung tissue of rats from the different groups.

\begin{tabular}{llll}
\hline Groups & IL-6 $(\mathrm{pg} / \mathrm{ml})$ & IL-8 $(\mathrm{pg} / \mathrm{ml})$ & IL-10 $(\mathrm{pg} / \mathrm{ml})$ \\
\hline LA & $30.09 \pm 2.77$ & $205.53 \pm 18.47$ & $173.95 \pm 7.69$ \\
MA1 & $53.65 \pm 3.97^{\mathrm{a}}$ & $285.10 \pm 29.36^{\mathrm{a}}$ & $199.50 \pm 38.24^{\mathrm{a}}$ \\
MA3 & $32.73 \pm 2.62$ & $207.81 \pm 23.91$ & $172.03 \pm 35.69$ \\
MA7 & $33.04 \pm 5.40$ & $205.03 \pm 28.40$ & $170.90 \pm 31.28$ \\
MA15 & $32.03 \pm 5.73$ & $203.08 \pm 24.09$ & $170.53 \pm 15.83$ \\
MA30 & $31.36 \pm 2.63$ & $206.85 \pm 15.41$ & $174.48 \pm 27.31$ \\
HA1 & $65.27 \pm 6.82^{\mathrm{a}}$ & $404.46 \pm 40.91^{\mathrm{a}}$ & $134.87 \pm 32.38^{\mathrm{a}}$ \\
HA3 & $58.76 \pm 6.91^{\mathrm{a}, \mathrm{b}}$ & $383.69 \pm 19.68^{\mathrm{a}}$ & $113.74 \pm 19.96^{\mathrm{a}}$ \\
HA7 & $51.13 \pm 5.72^{\mathrm{a}-\mathrm{c}}$ & $330.96 \pm 26.09^{\mathrm{a}-\mathrm{c}}$ & $97.88 \pm 11.68^{\mathrm{a}, \mathrm{b}}$ \\
HA15 & $43.37 \pm 5.34^{\mathrm{a}-\mathrm{d}}$ & $284.16 \pm 18.09^{\mathrm{a}-\mathrm{d}}$ & $99.17 \pm 3.73^{\mathrm{a}, \mathrm{b}}$ \\
HA30 & $37.63 \pm 3.52^{\mathrm{a}-\mathrm{e}}$ & $256.15 \pm 19.87^{\mathrm{a}-\mathrm{e}}$ & $96.91 \pm 14.55^{\mathrm{a}, \mathrm{b}}$
\end{tabular}

${ }^{\mathrm{a}-\mathrm{e}}$ Compared with the LA, HA1, HA3, HA7 and HA15 group, respectively. Data are the means $\pm \mathrm{SD}$ ( $\mathrm{n}=8$ per group). $\mathrm{P}<0.05$ for all. IL, interleukin; LA, low altitude; HA, MA, middle altitude; HA, high altitude; MA and HA1, 2, 7, 15, 30 represent the MA and HA subgroups at $1,3,7,15$ and 30 days, respectively.

from the rats in the different groups were stained with hematoxylin and eosin, and the morphological observations were observed under a microscope. Images of the pathological sections are shown in Fig. 1.

As regards the control group, the longitudinal plica mucosa in the terminal bronchioles was not obvious. In the respiratory bronchioles, the epithelial mucosa consisted of a simple columnar epithelium or a cuboidal epithelium, and the alveolar wall consisted of flat type I alveolar cells and cuboidal alveolar type II cells. The alveolar septum was rich in capillaries, 
Table III. Change in SOD and MDA levels in the lung tissue of rats from the different groups.

\begin{tabular}{llc}
\hline Groups & SOD (u/mgprot) & MDA (nmol/mgprot) \\
\hline LA & $439.85 \pm 33.80$ & $1.808 \pm 0.240$ \\
MA1 & $437.89 \pm 23.35$ & $1.824 \pm 0.251$ \\
MA3 & $438.86 \pm 26.74$ & $1.811 \pm 0.205$ \\
MA7 & $442.79 \pm 28.80$ & $1.820 \pm 0.197$ \\
MA15 & $441.70 \pm 15.21$ & $1.816 \pm 0.206$ \\
MA30 & $440.74 \pm 36.42$ & $1.813 \pm 0.354$ \\
HA1 & $554.07 \pm 24.59^{\mathrm{a}}$ & $2.386 \pm 0.345^{\mathrm{a}}$ \\
HA3 & $486.78 \pm 18.78^{\mathrm{a}, \mathrm{b}}$ & $2.993 \pm 0.340^{\mathrm{a}, \mathrm{b}}$ \\
HA7 & $403.71 \pm 16.57^{\mathrm{a}-\mathrm{c}}$ & $3.441 \pm 0.303^{\mathrm{a}-\mathrm{c}}$ \\
HA15 & $341.98 \pm 17.89^{\mathrm{a}-\mathrm{d}}$ & $4.033 \pm 0.876^{\mathrm{a}-\mathrm{d}}$ \\
HA30 & $295.98 \pm 11.08^{\mathrm{a}-\mathrm{e}}$ & $4.661 \pm 0.742^{\mathrm{a}-\mathrm{e}}$ \\
\hline
\end{tabular}

${ }^{\mathrm{a}-\mathrm{e}}$ Compared with the LA, HA1, HA3, HA7 and HA15 group, respectively. Data are the means $\pm \mathrm{SD}$ ( $\mathrm{n}=8$ per group). $\mathrm{P}<0.05$ for all. $\mathrm{SOD}$, superoxide dismutase; MDA, malondialdehyde. LA, low altitude; HA, MA, middle altitude; HA, high altitude; MA and HA1, 2, 7, 15, 30 represent the MA and HA subgroups at 1,3,7,15 and 30 days, respectively.

elastic fibers and macrophages, and the structure of the large and small pulmonary arteries was normal.

As regards the MA group, the longitudinal plica mucosa in the terminal bronchioles was evident. In the respiratory bronchioles, the epithelial mucosa consisted of a simple columnar epithelium or a simple cuboidal epithelium, and with the passing of time, due to capillary dilatation and congestion, the alveolar wall widened in part of the region, with focal alveolar hemorrhage, light thickening of the pulmonary artery and focal emphysema being observed.

As regards the HA group, the longitudinal plica mucosa in the terminal bronchioles was not obvious. In the respiratory bronchioles, the epithelial mucosa consisted of a columnar epithelium or a simple cuboidal epithelium, and due to capillary dilatation and congestion, the alveolar wall had widened in part of the region, with focal alveolar hemorrhage being observed. Focal alveolar hemorrhage and massive hemorrhage of the individual pulmonary tissue were also observed. The pulmonary small artery had slightly thickened. The wall of the larger arteriolar vessels showed focal thickening and there were a few lymphocytic infiltrations around the small blood vessels. With the passing of the time of exposure to hypobaric hypoxic conditions, damage to the structure of the lung tissue was observed, which gradually became more severe, as noted under a microscope.

\section{Discussion}

The results of this study demonstrated that the levels of IL-6/IL-8/IL-10 in the MA1 subgroup of the MA group were higher than those of the control group $(\mathrm{P}<0.05)$; however, no significant differences in these levels were observed among the other MA subgroups and the control group $(\mathrm{P}>0.05)$. The levels of IL-6/IL-8 in all the HA subgroups were higher compared to those of the control group $(\mathrm{P}<0.05)$, and even though these levels
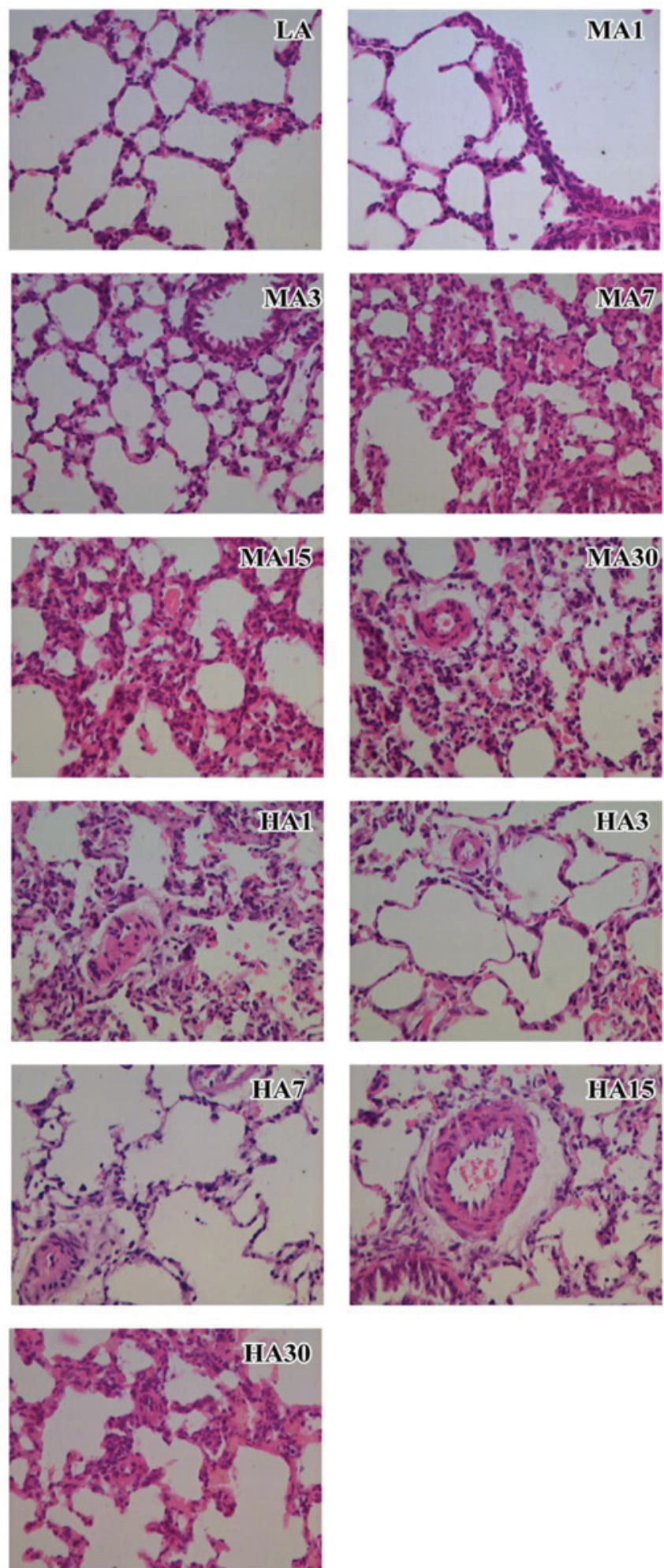

Figure 1. Images of the pathological observation of the lung tissues of the rats in the different groups (magnification, $\mathrm{x} 40$ ). See text for a detail description of the results in each group. LA, low altitude; HA, MA, middle altitude; HA, high altitude; MA and HA1, 2, 7, 15, 30 represent the MA and HA subgroups at $1,3,7,15$ and 30 days, respectively.

decreased with the passing of time, they were still higher than those of the control group $(\mathrm{P}<0.05)$. The levels of IL-10 decreased with the passing of time in the HA group, and were lower than those in the control group and the MA group $(\mathrm{P}<0.05)$. 
The biological effects of IL-6 involve the promotion of B lymphocyte proliferation and differentiation and the secretion of antibodies, functioning as endogenous pyrogens participating in inflammatory reactions, and enhancing the antitumor activity of cytotoxic $\mathrm{T}$ cells and natural killer cells (12). As an inflammatory cytokine, IL-6 has a number of biological effects, participating in the vascular endothelial inflammatory response, but also stimulating the production of inflammatory cytokines and increasing vascular inflammatory reactions $(13,14)$. IL-8 has strong chemotactic properties and activates neutrophil chemotaxis, as well as the chemotaxis of basophilic granulocytes, $\mathrm{T}$ lymphocytes and other inflammatory cells for angiogenesis $(15,16)$. On the other hand, IL-10 has an inhibitory effect on immune cell functions, and can prevent or inhibit specific and non-specific immune responses and the resulting damage, thus contributing to the induction of immune tolerance (17-19). Therefore, IL-6 and IL-8 are both pro-inflammatory cytokines, and participate in the inflammatory response and angiogenesis. IL-10 is an inhibitory inflammation factor, and can reduce tissue damage and promote the process immune tolerance.

In this study, since the levels of IL-6/IL-8/IL-10 in the MA1 group were higher than those of the control group $(\mathrm{P}<0.05)$, and no significant differences in these levels were observed among the other MA subgroups and the control group, this indicates that exposure to mid-altitude environment increases the inflammatory reaction in the lung tissue of rats. As the antiinflammatory effect took place, the levels of IL-6/IL-8/IL-10 quickly returned to levels close to the basal (control) levels after 3 days, indicating that the altitude conditions did not have a long-term effect on airway inflammatory reactions in the lung tissue. In the HA group however, with the passing of time, the IL-6/IL-8 levels gradually decreased following a sudden increase on day 1 , but were still higher than those of the control group, indicating that the inflammatory reaction resulting from hypoxia decreased with the passing of time; this indicates the adaptation process to hypoxic conditions. However, the inflammation state induced by exposure to hypoxia persisted, and injury due to inflammation was sustained. The level of IL-10 decreased under hypoxic conditions, indicating that hypoxia leads to a decrease in the ability to inhibit immunity and inflammation. Therefore, IL-10 cannot prevent and suppress strong specific and non-specific immune responses and the resulting damage, as well as reduce immune tolerance. This explains why a high altitude hypoxic environment causes airway inflammation of the lung tissue and injury to a certain extent.

The results of this study demonstrated that there were no significant differences in SOD activity and the MDA content between all MA subgroups and the control group. With the passing of time, SOD activity decreased and the MDA content gradually increased in all the HA subgroups $(\mathrm{P}<0.05)$. SOD is an antioxidant and can protect the cells of the body from oxidative damage $(5,20)$. Ischemia and hypoxia inhibit SOD activity, weakening its antioxidant abilities.

MDA is a stable metabolite of lipid peroxidation, and reflects the free radical content and the degree of lipid peroxidation, and indirectly reflects the degree of cell injury $(6,21)$. The results of this study demonstrated that there were no differences in SOD activity and the MDA content between the MA group and the control group, suggesting that the antioxidant activity and lipid peroxidation in the lung tissue were not altered, and there is no obvious cell injury in the lung tissue. However, in the HA group, the activity of SOD in all the subgroups was higher than that of the control group, and with the passing of time it gradually decreased to levels lower than those of the control group. This indicates that following initially entry into a high altitude environment, the lung tissue antioxidant capacity is increased, so that the cells of the body become immune to oxidative damage. However, with prolonged exposure to low oxygen, the increasing consumption of SOD causes rapid oxidative damage and an ongoing development of oxidative cellular damage. In the HA group, since the MDA content gradually increased with the passing of time, this further confirmed that the content of free radicals in the lung tissue increased, resulting in a greater degree of lipid peroxidation and severe damage to lung tissue. This explains why a high altitude hypoxic environment causes oxidative damage to lung tissue to a certain extent.

Subsequently, through the observation of the pathomorphism of the lung tissue of the rats in each group, we found that compared to the low altitude control group, there was a gradual and mild damage to the lung tissue of the rats in the MA group with the prolongation of the exposure time, but no inflammatory cell infiltration. However, in the HA group, with the extension of the time of exposure to hypoxia, lung tissue damage in the rats was aggravated, which is consistent with the changes in interleukin, SOD and MDA levels in lung tissue.

Therefore, the results of this study suggest that high altitude hypoxia induces lung inflammation and progressive oxidative damage which causes a serious degree of damage to lung tissue and the weakening of the inhibition of the inflammatory response.

\section{Acknowledgements}

This study was funded by Foundation Research Project of Qinghai Science and Technology Department (no. 2013-Z-741).

\section{References}

1. Opal SM and DePalo VA: Anti-inflammatory cytokines. Chest 117: 1162-1172, 2000.

2. Li A, Dubey S, Varney ML, Dave BJ and Singh RK: IL-8 directly enhanced endothelial cell survival, proliferation, and matrix metalloproteinases production and regulated angiogenesis. J Immunol 170: 3369-3376, 2003.

3. Hilgenberg E, Shen P, Dang VD, Ries S, Sakwa I and Fillatreau S: Interleukin-10-producing B cells and the regulation of immunity. Curr Top Microbiol Immunol 380: 69-92, 2014.

4. Hofmann SR, Rösen-Wolff A, Tsokos GC and Hedrich CM: Biological properties and regulation of IL-10 related cytokines and their contribution to autoimmune disease and tissue injury. Clin Immunol 143: 116-127, 2012.

5. Seyhan $N$ and Canseven AG: In vivo effects of ELF MFs on collagen synthesis, free radical processes, natural antioxidant system, respiratory burst system, immune system activities, and electrolytes in the skin, plasma, spleen, lung, kidney, and brain tissues. Electromagn Biol Med 25: 291-305, 2006.

6. Niki E: Lipid peroxidation products as oxidative stress biomarkers. Biofactors 34: 171-180, 2008.

7. Sarada S, Himadri P, Mishra C, Geetali P, Ram MS and Ilavazhagan G: Role of oxidative stress and NFkB in hypoxiainduced pulmonary edema. Exp Biol Med (Maywood) 233: 1088-1098, 2008.

8. Kubo K, Hanaoka M, Yamaguchi S, Hayano T, Hayasaka M, Koizumi T, Fujimoto K, Kobayashi T and Honda T: Cytokines in bronchoalveolar lavage fluid in patients with high altitude pulmonary oedema at moderate altitude in Japan. Thorax 51: 739-742, 1996. 
9. Heffner JE and Repine JE: Pulmonary strategies of antioxidant defense. Am Rev Respir Dis 140: 531-554, 1989.

10. Bulger EM and Maier RV: Antioxidants in critical illness. Arch Surg 136: 1201-1207, 2001.

11. Nakanishi K, Tajima F, Nakamura A, Yagura S, Ookawara T, Yamashita H, Suzuki K, Taniguchi N, Ohno H: Effects of hypobaric hypoxia on antioxidant enzymes in rats. J Physiol 489: 869-876, 1995.

12. Diehl S and Rincón M: The two faces of IL- 6 on Th1/Th2 differentiation. Mol Immunol 39: 531-536, 2002.

13. Packard RR and Libby P: Inflammation in atherosclerosis: From vascular biology to biomarker discovery and risk prediction. Clin Chem 54: 24-38, 2008.

14. Akira S, Taga T and Kishimoto T: Interleukin-6 in biology and medicine. Adv Immunol 54: 1-78, 1993

15. Remick DG: Interleukin-8. Crit Care Med 33 (Suppl 12): S466-S467, 2005.

16. Waugh DJ and Wilson C: The interleukin-8 pathway in cancer. Clin Cancer Res 14: 6735-6741, 2008.
17. Delogu G, Antonucci A, Signore M, Marandola M, Tellan G and Ippoliti F: Plasma levels of IL-10 and nitric oxide under two different anaesthesia regimens. Eur J Anaesthesiol 22: 462-466, 2005.

18. Miller BJ, Buckley P, Seabolt W, Mellor A and Kirkpatrick B Meta-analysis of cytokine alterations in schizophrenia: Clinical status and antipsychotic effects. Biol Psychiatry 70: 663-671, 2011.

19. Moore KW, de Waal Malefyt R, Coffman RL and O'Garra A: Interleukin-10 and the interleukin-10 receptor. Annu Rev Immunol 19: 683-765, 2001.

20. Suzer T, Coskun E, Demir S and Tahta K: Lipid peroxidation and glutathione levels after cortical injection of ferric chloride in rats: effect of trimetazidine and deferoxamine. Res Exp Med (Berl) 199: 223-229, 2000.

21. Nielsen F, Mikkelsen BB, Nielsen JB, Andersen HR and Grandjean P: Plasma malondialdehyde as biomarker for oxidative stress: reference interval and effects of life-style factors. Clin Chem 43: 1209-1214, 1997. 